seen as very simplistic, often just 'food lists' with little or no personalisation to meet individual needs of the participants (figure 1). Digital online and resources were used to supplement the dietary information received form GP's and GE's, however this required additional interpretation and personalisation and led to negative effects on both the participants social and food-related quality of life.

Conclusion The participants found much of the nutritional information provide by GPS' and GE's to be overly generic and incomplete; in that it was difficult to apply in 'real life'. The findings in this study support the current clinical guidelines proposed by the both by NICE and the BDA that LFD and GFD's should still be considered second-line dietitian-led only interventions.

\section{PWE-125 EFFECT OF TURMERIC ON THE FAECAL VOLATILE ORGANIC METABOLITES IN HEALTHY INDIVIDUALS}

Gaurav Vashisht*, Kantida Koysombat, Rachael Hough, Lauren Lett, Sherry Browne, Naomi Lloyd, Chris Probert. University Of Liverpool, Liverpool, UK

\subsection{6/gutjnl-2018-BSGAbstracts.359}

Introduction Inflammatory bowel disease (IBD) affects the gut microbiome and metabolome. Curcumin, from turmeric, may be of benefit in some patients through action on the gut microbiome. Curcumin produces changes to bile acid secretion, and has a variety of direct effects on bacteria. Faeces release Faecal Volatile Organic Metabolites (VOMs), which partly reflect the gut microbiome. The aim of the study was to investigate the effects of turmeric on the VOMs in healthy individuals.

Methods 5 participants were enrolled in a before-during-after pilot study, in which they were asked to take a turmeric-free diet and then to consume $1.6 \mathrm{~g}$ of turmeric daily for 5 days. Faecal samples were collected at baseline, after 5 days of turmeric ingestion, and again 5 days after this, and frozen immediately. The samples were analysed by an investigator blinded using gas chromatography mass spectrometry. Analytes were identified using AMDIS software and compared using Metaboanalyst software: ANOVA, PCA, PLSADA, and Heatmap were employed.

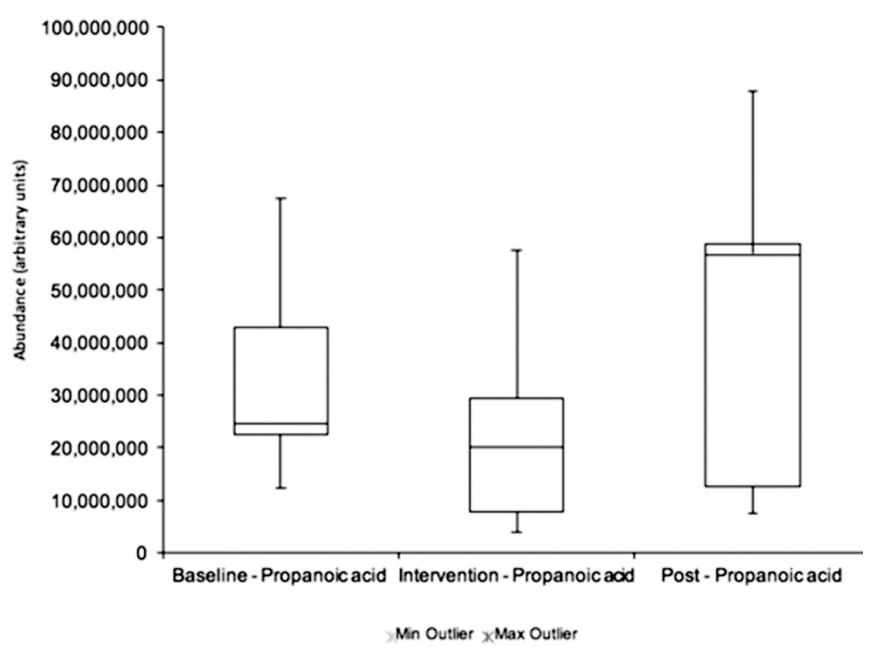

Abstract PWE-125 Figure 1 Box plot showing changes in abundance of propanoic acid amongst the comparison groups

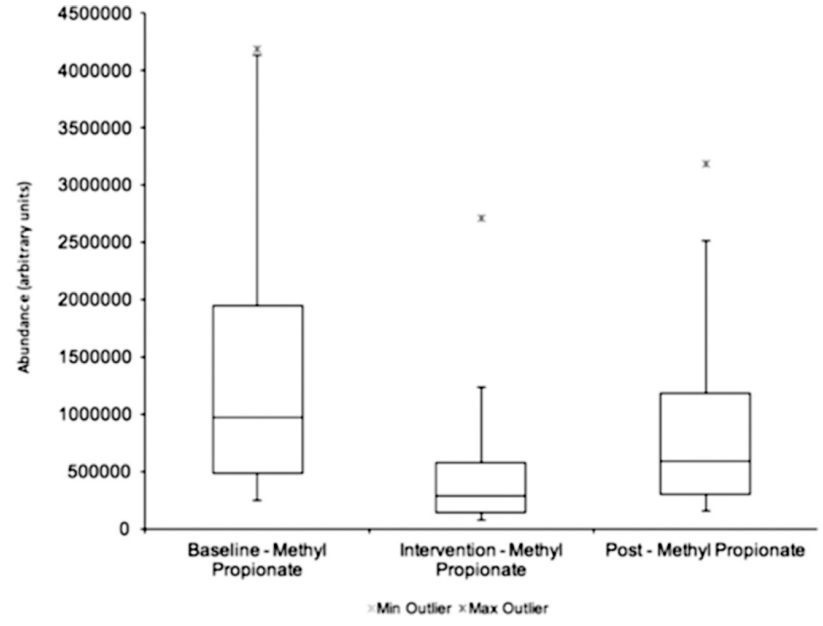

Abstract PWE-125 Figure 2 Box plot showing changes in abundance of methyl propionate amongst the comparison groups

Results ANOVA yielded 0 significant features. For most of the VOMs found between the comparison groups, $p>0.05$. Both PCA and PLSADA failed to show any separation by group. Heatmap analysis did not show any pattern in the abundance of VOMs. VIP scores showed a decrease in the abundance of propanoic acid and methyl propionate in the intervention samples when compared to baseline and post-turmeric consumption. The box plots created from raw data in Figure $1 \& 2$ demonstrated lower median abundances of intervention samples as compared to comparison groups. The study was underpowered to demonstrate significant change.

Conclusions This pilot study illustrates that two VOMs appears to become less abundant when turmeric is consumed: both appear related to propionibacteria metabolism. Studies in patients with IBD are warranted.

\section{PWE-126 LOW FODMAP DIET EFFECT ON IBS GASTROINTESTINAL MICROBIOME AND METABOLITES AND PREDICTION OF RESPONSE}

${ }^{1}$ Bridgette Wilson*, ${ }^{1}$ Megan Rossi, ${ }^{1}$ Tokuwa Kanno, ${ }^{2}$ Rachael Hough, ${ }^{2}$ Chris Probert, 1,3Peter Irving, ${ }^{1}$ A James Mason, ${ }^{1,3}$ Miranda Lomer, 'Kevin Whelan*. 'King's College London, London, UK; ${ }^{2}$ University of Liverpool, Liverpool, UK; ${ }^{3}$ Guy's and St Thomas' NHS Foundation Trust, London, UK

\subsection{6/gutjnl-2018-BSGAbstracts.360}

Introduction Prebiotic $\beta$-galactooligosaccharides (B-GOS) may counteract the microbiome modifying effect of the low FODMAP diet (LFD) in patients with irritable bowel syndrome (IBS). Faecal metabolites may predict why only some patients respond to the LFD paving the way towards more personalised treatment.

The aim of this randomised controlled trial (RCT) was to investigate: a) the impact of the LFD and LFD $+1.4 \mathrm{~g} / \mathrm{d}$ BGOS compared to Control on the gut microbiome in IBS, and b) if differences in faecal or urinary metabolites predict response to the LFD.

Methods A 3-arm RCT was performed in 69 IBS patients randomised to: Sham diet +Placebo (Control), LFD + Placebo (LFD) or LFD +B GOS. This study investigated global 